\title{
The Dark Energy Survey and Operations: Year 6 - The Finale
}

\author{
H. T. Diehl ${ }^{\text {la }}$, B. Yanny ${ }^{\mathrm{a}}$, D. L. Tucker ${ }^{\mathrm{a}}$, F. Paz-Chinchón ${ }^{\mathrm{b}}$, E. Neilsen ${ }^{\mathrm{a}}$, S. Kent ${ }^{\mathrm{a}}$,
} M. D. Johnson ${ }^{\mathrm{b}}$, R. A. Gruendl ${ }^{\mathrm{b}, \mathrm{c}}$, Y.-C. Chen ${ }^{\mathrm{c}}$, Z. Zhang $^{\mathrm{d}}$, Y. Zhang ${ }^{\mathrm{a}}$, A. Zenteno ${ }^{\mathrm{e}}$, R. Wilkinson ${ }^{\mathrm{f}}$, N. Weaverdyck ${ }^{\mathrm{g}}$, Q. Wang ${ }^{\mathrm{d}}$, A. R. Walkere, E. Wagoner ${ }^{\mathrm{h}}$, A. K. Vivas ${ }^{\mathrm{e}}$,

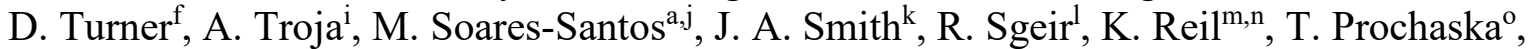
A. A. Plazas ${ }^{\mathrm{p}}$, D. Petravick ${ }^{\mathrm{b}}$, A. Palmese ${ }^{\mathrm{a}}$, M. Murphy ${ }^{\mathrm{a}}$, J. Muir ${ }^{\mathrm{m}}$, R. Morgan ${ }^{\mathrm{q}}$, P. Martini ${ }^{\mathrm{r}, \mathrm{s}}$, C. E. Martinez-Vazquez ${ }^{\mathrm{e}}$, L. Markwardt ${ }^{\mathrm{g}}$, J. L. Marshallo ${ }^{\mathrm{o}}$, T. S. Li ${ }^{\mathrm{a}}$, A. Lathrop ${ }^{\mathrm{a}}$, N. Kuropatkin ${ }^{\mathrm{a}}$, R. Kron $^{\mathrm{a}, \mathrm{d}}$, M. R. Kirby ${ }^{\mathrm{h}}$, D. J. James ${ }^{\mathrm{t}}$, B. Jain ${ }^{\mathrm{u}}$, T. A. Hutchinson ${ }^{\mathrm{o}}$, K. Honscheid ${ }^{\mathrm{r}, \mathrm{v}}$, D. Hollowood ${ }^{\mathrm{w}}$, K. Herner ${ }^{\mathrm{a}}$, D. Gruen ${ }^{\mathrm{m}, \mathrm{n}}$, M. S. S. Gill ${ }^{\mathrm{n}}$, A. Garcia ${ }^{\mathrm{j}}$, B. Flaugher ${ }^{\mathrm{a}}$, J. Frieman ${ }^{\mathrm{a}, \mathrm{m}, \mathrm{d}}$, S. Everett ${ }^{\mathrm{w}}$, J. DeRose ${ }^{\mathrm{m}, \mathrm{n}, \mathrm{x}}$, D. Huterer ${ }^{\mathrm{g}}$, L. da Costa $^{\mathrm{i}}$, A. Chen ${ }^{\mathrm{g}}$, A. Campos $^{\mathrm{y}}$, G. M. Bernstein ${ }^{\mathrm{u}}$, K. Bechtol ${ }^{\mathrm{q}, \mathrm{z}}$,

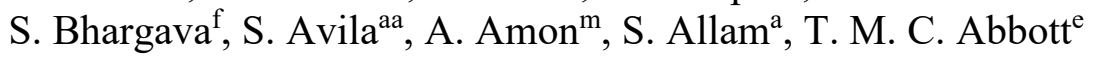

${ }^{\mathrm{a}}$ Fermi National Accelerator Laboratory, P.O. Box 500, Batavia, IL 60510, USA; ${ }^{\mathrm{b}}$ National Center for Supercomputing Applications, 1205 West Clark St., Urbana, IL 61801, USA; ${ }^{c}$ Department of Astronomy, University of Illinois at Urbana-Champaign, W. Green Street, Urbana, IL 61801, USA; 'Department of Astronomy and Astrophysics, University of Chicago,

Chicago, IL 60637, USA; 'National Optical Astronomy Observatory, Cerro Tololo InterAmerican Observatory, Casilla 603, La Serena, Chile; ' Department of Physics and Astronomy, Sussex, UK; ' ${ }^{D}$ Department of Physics, University of Michigan, Ann Arbor, MI 48109, USA;

hDept. of Physics, University of Arizona, Tucson, AZ 85721, USA; 'iLaboratório

Interinstitucional de e-Astronomia - LIneA, Rua Gal. José Cristino 77, Rio de Janeiro, RJ 20921-400, Brazil; 'ंDepartment of Physics, Brandeis University, Waltham, MA 02453, USA; kDepartment of Physics and Astronomy, Austin Peay State University, Clarksville, TN 37044, USA; ' Institute for Astronomy, Department of Physics, ETH Zurich, Wolfgang-Pauli-Strasse 27, CH-8093 Zurich, Switzerland; 'Mavli Institute for Particle Astrophysics and Cosmology, 452

Lomita Mall, Stanford University, Stanford, CA 94305, USA; " SLAC National Accelerator Laboratory, Menlo Park, CA 94025, USA; ${ }^{\circ}$ George P. and Cynthia Woods Mitchell Institute for Fundamental Physics and Astronomy, and Department of Physics and Astronomy, Texas A\&M University, College Station, TX 77843, USA; ${ }^{\mathrm{p} D e p a r t m e n t ~ o f ~ A s t r o p h y s i c a l ~ S c i e n c e s, ~ P e y t o n ~}$ Hall, Princeton University, Princeton, NJ 08544, USA; ${ }^{9}$ Physics Department, University of Wisconsin-Madison, 1150 University Avenue Madison, WI 53706-1390, USA; ${ }^{\mathrm{r} C e n t e r}$ for Cosmology and Astro-Particle Physics, The Ohio State University, Columbus, OH 43210, USA; ${ }^{\mathrm{s}}$ Department of Astronomy, The Ohio State University, Columbus, OH 43210, USA; 'HarvardSmithsonian Center for Astrophysics, Cambridge, MA 02138, USA; "Department of Physics and Astronomy, University of Pennsylvania, Philadelphia, PA 19104, USA; 'Department of Physics, The Ohio State University, Columbus, OH 43210, USA; "wepartment of Physics and Santa Cruz Institute for Particle Physics, University of California, Santa Cruz, CA 95064, USA; xDepartment of Physics, Stanford University, 382 Via Pueblo Mall, Stanford, CA 94305, USA; ${ }^{y}$ Department of Physics, Carnegie Mellon University, Pittsburgh, Pennsylvania 15312, USA;

${ }^{z} L S S T, 933$ North Cherry Avenue, Tucson, AZ 85721, USA; ${ }^{a a}$ Instituto de Fisica Teorica UAM/CSIC, Universidad Autonoma de Madrid, 28049 Madrid, Spain

\footnotetext{
${ }^{1}$ Diehl@FNAL.GOV; phone 1-630-840-8307.
} 


\begin{abstract}
The Dark Energy Survey (DES) is an optical and near-infrared imaging survey aimed at understanding the accelerating expansion of the universe using four complementary methods: weak gravitational lensing, galaxy cluster counts, baryon acoustic oscillations, and Type Ia supernovae. To perform the 6-season 5000 sq-degree wide field and the 5season 30 sq-degree supernova surveys, the DES Collaboration built the Dark Energy Camera (DECam), a 3 squaredegree, 570-Megapixel CCD camera that was installed at the prime focus of the Blanco 4-meter telescope at the Cerro Tololo Inter-American Observatory (CTIO). DES started its observations in "Year 1" (Y1) on Aug. 31, 2013 and completed its sixth and final observing season on January 9, 2019. This paper describes DES Y6, the survey strategy, an outline of the survey operations procedures, the efficiency of operations and the causes of lost observing time. It provides details about the quality of the final season's data, and a summary of the Y1-Y6 observing. It contains a brief description of the last night of observations.
\end{abstract}

Keywords: Cosmology, Dark Energy Survey, Dark Energy Camera, Operations, CTIO

\title{
1. INTRODUCTION
}

The Dark Energy Survey (DES) team is an international collaboration, with over 700 scientists from 25 institutions and consortiums in the US, Chile, the UK, Spain, Brazil, Switzerland, and Germany. The DES [1] main science goal is to measure dark energy parameters using four complementary techniques: galaxy cluster counting, baryon acoustic oscillations, weak gravitational lensing, and Type Ia supernovae. A rich dataset also enables Milky Way, solar system, and a variety of other extragalactic science [2]. DES comprises two interleaved surveys. The wide-field (WF) survey identifies stars and galaxies, and reports shapes and magnitudes in 5 filters, in 5100 square degrees of the southern galactic cap. A transient survey repeatedly images ten 2.7-square-degree fields with the purpose of identifying Type 1a supernovae. To carry out these surveys, the DES Collaboration constructed a new instrument, the Dark Energy Camera and installed it on the Victor M. Blanco 4m telescope on Cerro Tololo in Chile during 2012. A "Science Verification" period followed over the next few months. After that, DES carried out survey operations, yearly, during six seasons. The first such season, called Y1, started on Aug. 31, 2013 and finished on Feb. 9, 2014. DES observed for 105 full-night equivalents. Four subsequent 105 night observing seasons [3-5], spanning roughly mid-August to mid-February, were called Y2, Y3, Y4 and Y5. The final observing season, Y6, comprised 52 full-night equivalents for DES. It started on Sept. 8, 2018 and ended on Jan. 9, 2019. There are no plans for another DES observing season. The Dark Energy Camera will continue to be operated as a community instrument by CTIO. This note describes Y6 operations.

\section{The Dark Energy Camera and Auxiliary Detectors}

The Dark Energy Camera (DECam) [6] consists of a wide-field corrector, a mosaic CCD imager and associated mechanical, optical, and electronic components. The optical corrector has 5 fusedsilica optical elements to attain an $\mathrm{f} / 2.7,2$-degree-wide image at the focal plane. The DES uses primarily five filters: DES g, r, i, z, and Y-bands, with central wavelengths 473, 642, 784, 926, and $1009 \mathrm{~nm}$, respectively. The focal plane itself has a $42-\mathrm{cm}$ radius and is populated with sixtytwo (62) 2048x4096 pixel, $250 \mu \mathrm{m}$ thick, fully-depleted, red-sensitive CCDs for imaging as well as $122048 \times 2048$ pixel CCDs for guiding and focus. Figure 1 shows a photo of the Dark Energy Camera mounted at the prime focus of the Blanco telescope.

Three auxiliary detectors on the CTIO summit, supplied by DES, provide information for photometric calibration. An All-Sky Radiometric Camera (RASICAM) [6-8] is used to monitor 
the sky using the wavelength range $970 \mathrm{~nm}$ to $1250 \mathrm{~nm}$. In this wavelength range, relatively warm clouds are easily distinguished from cold, clear skies. The Atmospheric Transmission Monitoring Camera (aTmCam) [6,9-10] has been operated consistently starting at the beginning of Y2. The aTmCam consists of a Paramount telescope mount and four small telescopes, each with a different narrowband filter, which monitors the brightness of suitable standard stars, thus providing the atmospheric transmission in wavelength regions dominated by the PWV and aerosol optical depth.

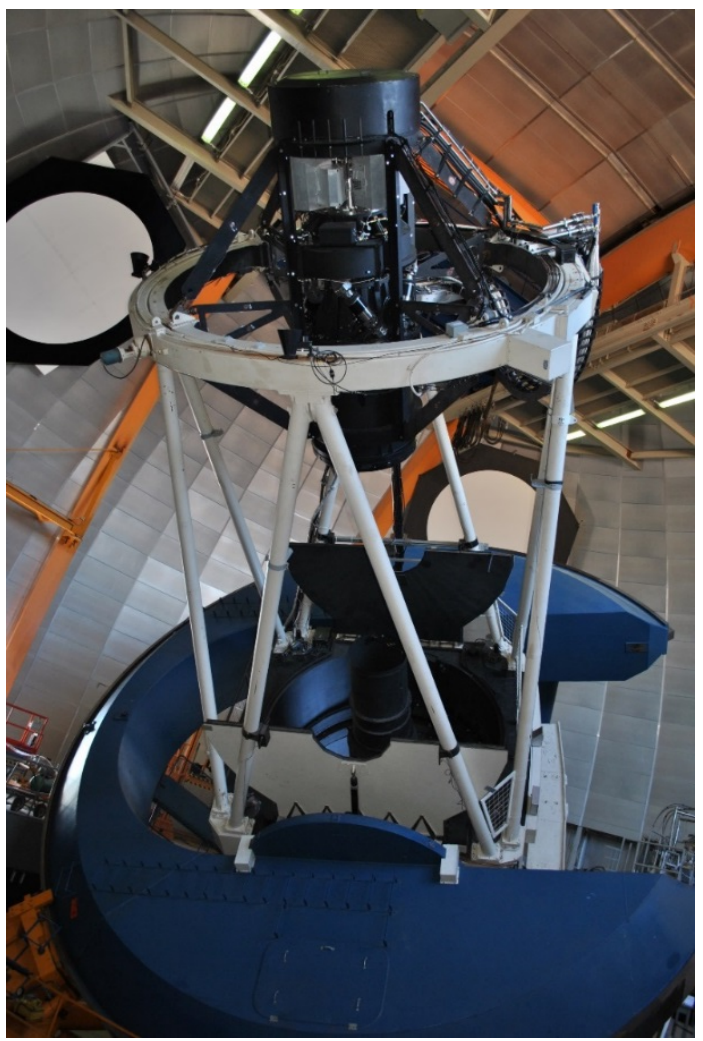

Figure 1 The Dark Energy Camera is mounted at the prime focus of the Victor M. Blanco 4m telescope at CTIO. The covers for the primary mirror are open. The camera assembly, including the support cage, is approximately $3.6 \mathrm{~m}$ long and is secured to the inner telescope ring. The camera, not including the support cage and counterweights, weighs approximately $4350 \mathrm{~kg}$. Subsequent to the date of this photo, the cage was retrofitted with aluminum sidecovers. The aluminum covers of two of the four readout electronics crates are visible near the top of the camera, just underneath the "Top Cap".

\section{The Dark Energy Survey Fields \& Footprint}

The 5000 square-degree WF survey has three main regions (see Figure 2) within the southern Galactic Cap. There is a broad roughly circular region from RA of roughly 0 to 120 degrees and DEC -70 to -10 degrees that provides a large contiguous area for the large-scale structure measurements. There is a wide roughly box-shaped region around the South Pole Telescope (SPT) observing area [11]. Finally, the survey encompasses a part of SDSS Stripe 82 [12], primarily for photometric calibration purposes. The ten 2.7 square-degree transient fields are observed with a cadence of about 7 days throughout the DES seasons, which are about $1 / 2$-year long. 
The footprint of a single DECam exposure is roughly hexagonal, constrained to an orientation aligned with celestial coordinates by the equatorial mount of the Blanco telescope; therefore, an area of the sky covering a particular range of R.A. can be covered with minimal gaps and overlaps by using a hexagonal tiling pattern aligned in declination. A "tiling" is a set of exposures, one in each of 5 filters, at pointings arranged in such a pattern. Over wider ranges of R.A. the planar approximation of the sky breaks down, so the strictly hexagonal layout is "broken" every 30 degrees of R.A., resulting in extra overlaps between exposures within a tiling at 30 degree intervals. A single tiling collects useful science data on roughly $83 \%$ of the footprint area due to these breaks, deviation from the planar approximation with each 30-degree R.A. neighborhood, and incomplete coverage within each hex (due to, for example, gaps between CCDs, bad CCDs, and problematic area near the edges of the CCDs). The plan for the DES WF survey specifies 10 separate tilings, each offset from the others by a significant fraction of the camera field of view, such that observations of individual astronomical sources are spread across the focal plane. The $g$, $\mathrm{r}, \mathrm{i}$, and z-band exposures are 90 seconds duration. The Y-band exposures are 45 seconds duration for tilings 1-6. In Y4 we changed the Y-band observations to 90 seconds for tilings 7 and 9 and dropped tilings 8 and 10 to improve the observing efficiency.

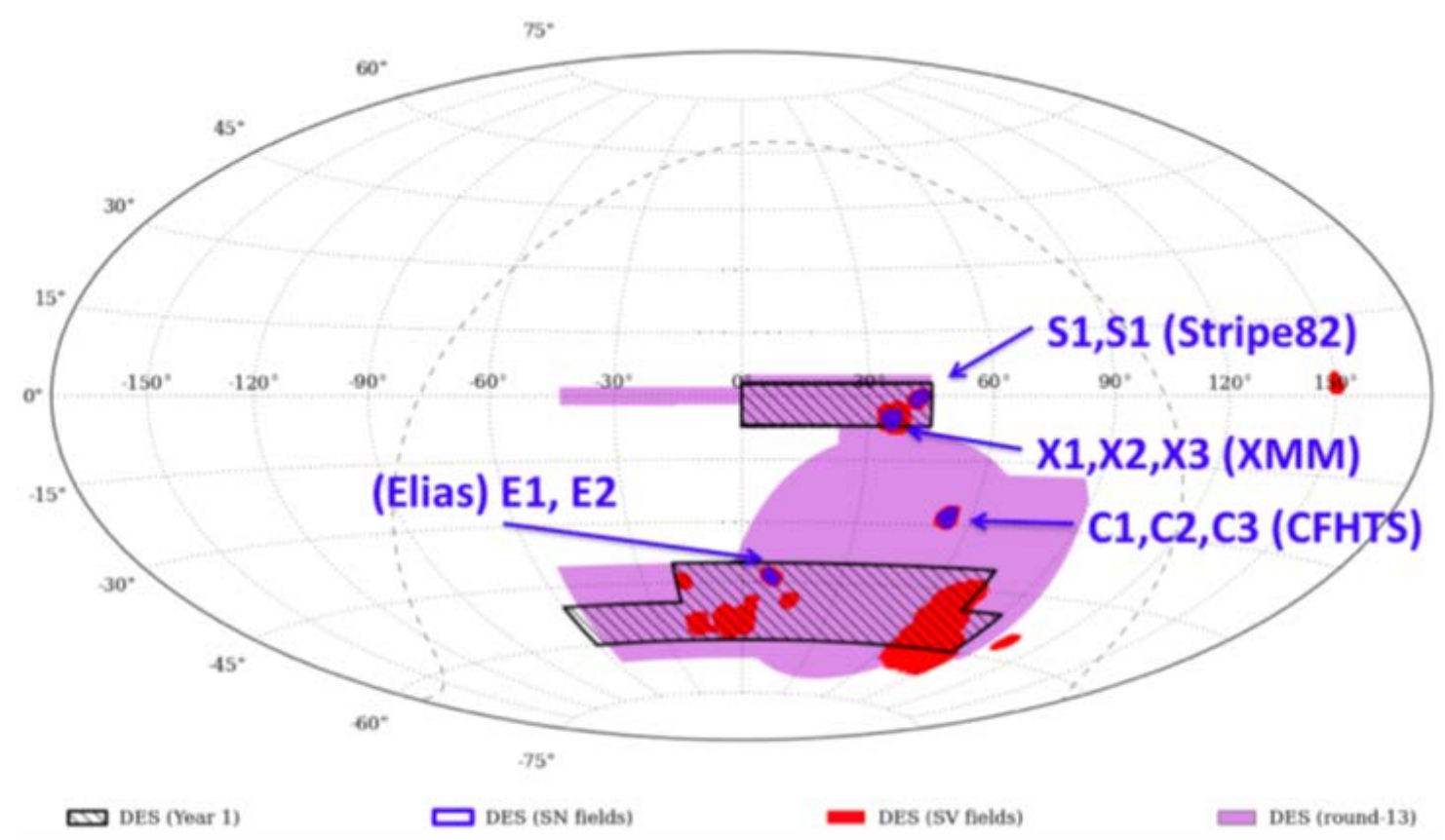

Figure 2. The Dark Energy Survey observing fields shown on this plot of RA and DEC. The Y1-Y6 wide field survey area is shaded light-purple. During "Science Verification" (Nov. 2012 - Feb. 2013), we observed the fields that are shaded red. The 10 transient (SN) fields observed in Y1 to Y5 are shaded in dark blue. The SV fields that overlap the Y1-Y6 wide field survey and the SN fields were also observed as part of the wide field survey. During Y1 (Aug. 2013 - Feb. 2014), DES observed the areas outlined in dark blue, which encompass SDSS Stripe 82 (upper) and the South Pole Telescope region (lower). In Y2 we observed the complementary part of the WF. After that we aimed for two complete tilings per season for Y3 to Y5. In Y6 we had roughly one tiling left to do to complete the 10-tiling survey.

We include a description of the Y1 to Y5 time domain survey as a matter of completeness, for we didn't execute it during Y6. The time domain survey was used to identify Type Ia SNe through 
difference imaging [13] and measurement of the light curves. The 10 time-domain fields, shown in Table 1, were observed on a regular cadence. The 8 "shallow fields" are observed for single exposures in g-band (175s), r-band (150s), and i-band (200s) and for two images in z-band (200s each). The 5 exposures of a shallow field were considered a "sequence" and the sequence is observed consecutively. The two "deep fields" were observed for 3 exposures of 200 s each in gband, for 400s each in r-band, for 5 exposures of 360s each in i-band, and for 11 exposures of 330s each in z-band. The exposures in each given filter for the deep fields were considered a sequence. For each filter, those exposures were sequential, but the different filters might be observed at different times during a single night or on different nights (deep field only). The limiting magnitudes [13] for difference imaging detections was about 23.5 in each band of the shallow field epochs and about 24.5 for the deep field epochs.

\begin{tabular}{|c|c|c|}
\hline Field Name & RA & DEC \\
\hline E1 & $7.87440(00: 31: 29.86)$ & $-43.0096(-43: 00: 34.6)$ \\
\hline E2 & $9.50000(00: 38: 00.00)$ & $-43.9980(-43: 59: 52.8)$ \\
\hline S1 & $42.82000(02: 51: 16.80)$ & $0.0000(00: 00: 00.0)$ \\
\hline S2 & $41.19440(02: 44: 46.66)$ & $-0.9884(-00: 59: 18.2)$ \\
\hline C1 & $54.27430(03: 37: 05.83)$ & $-27.1116(-27: 06: 41.8)$ \\
\hline C2 & $54.27430(03: 37: 05.83)$ & $-29.0884(-29: 05: 18.2)$ \\
\hline C3 & $52.64840(03: 30: 35.62)$ & $-28.1000(-28: 06: 00.0)$ \\
\hline X1 & $34.47570(02: 17: 54.17)$ & $-4.9295(-04: 55: 46.2)$ \\
\hline X2 & $35.66450(02: 22: 39.48)$ & $-6.4121(-06: 24: 43.6)$ \\
\hline X3 & $36.45000(02: 25: 48.00)$ & $-4.6000(-04: 36: 00.0)$ \\
\hline
\end{tabular}

Table 1 RA and DEC (J2000) of the 10 DES supernova fields. Fields C3 and X3 are "deep fields". The other 8 fields are "shallow fields". We didn't execute the SN survey during Y6.

This paper describes DES "Year 6" (Y6). Section 2 describes the maintenance and improvements to the camera and observing systems. Section 3 describes the survey procedures. Section 4, the Y6 and narrative including a description of the strategy and goals for the data-taking, the efficiency of survey operations and the progress towards the survey goals. Some information summarizing the Y1 - Y6 observations is mixed into this section. Section 5 describes the last night of DES observations. Section 6 provides a terse summary.

\section{CAMERA \& TELESCOPE MAINTENANCE AND UPGRADES: Y6}

Generally, the camera and telescope performance has been very good since Science Verification, which was during 2012. A continuous effort to improve has been carried-out by scientists and technical staff from CTIO and DES. We discussed those that occurred in 2012 to 2017 in the "DECam Paper" [6] and elsewhere [3-5]. There are a few items that we discuss in some detail here. The first is routine maintenance to the focal plane CCD LN2-based cooling system. Next, we describe the condition of the CCDs through Y6. After that, the maintenance on the shutter and filter changer. Finally, we describe the results of CTIO's resurfacing of the DECam primary mirror. 


\section{Maintenance of the CCD Cooling System}

The DECam focal plane is kept at operating temperature, $-100 \mathrm{C}$, by means of a liquid nitrogen $\left(\mathrm{LN}_{2}\right)$ system. A 200L tank resides on a platform in the dome, just to the side of the telescope. A pump, submerged in the tank, pumps 2 gallons of $\mathrm{LN}_{2}$ per minute to a heat exchanger, connected

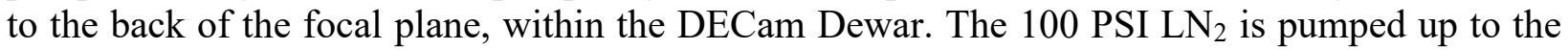
camera and returned in vacuum-jacketed pipes with total length of $\sim 470$ feet. The total heat-load should be $<500 \mathrm{~W}$. The heat goes into converting $\mathrm{LN}_{2}$ to gaseous $\mathrm{N}_{2}$. Two $300 \mathrm{~W}$ compressed-He cryocoolers supply the refrigeration that re-liquifies the two-phase $\mathrm{N}_{2}$ that is returned to the tank. For more details, see the DECam paper [6]. Because the cryo-coolers can supply refrigeration greater than the heat load, it is operable as a closed system. In the circumstance that the heat load is larger than the cooling capability, the $\mathrm{N}_{2}$ gas pressure builds until it is vented and the $\mathrm{N}_{2}$ lost. Up to the end of Y4 we had not sustained closed-loop operation for long periods. The principal contributor to the extra heat load was a leak from one of the high-pressure N2 pipes into the vacuum jackets for the section of pipe that connected to the imager Dewar. The Teflon gasket near the camera was replaced in Sept. 2017 and the system has been in closed-loop operation since. There is a second issue with the $\mathrm{LN}_{2}$ system. Discussed in detail in [6], the problem is the bearing cages that support the rotor shaft in the $\mathrm{LN}_{2}$ pump wear away. That produces a continued need to periodically \& proactively replace the submerged $\mathrm{LN}_{2}$ pump. That was done in March 2018, shortly after the end of Y5, and again in mid-November 2018, in a Full Moon period during Y6. CTIO was trained in the procedure for the replacement of the bearings.

The arrangement of the DECam focal plane, with $622 \mathrm{kx} 4 \mathrm{k}$ imaging CCDs, $42 \mathrm{kx} 2 \mathrm{k}$ "Guide" CCDs and 8 2kx2k "alignment" CCDs, is shown in Figure 3. These are in good working order except for CCD N30, which failed Nov. 7, 2012. Amplifier A of CCD S7 has an unpredictable time-varying gain and is not used for DES science. CCD S30 failed on Nov. 30, 2013 but, oddly, returned to good condition Dec. 29, 2016, 37 months later. We presume some material in the Dewar that had caused an amplifier ( $\left.\mathrm{V}_{\text {reset }}\right)$ short simply fell off. On Nov. 10, 2017, CCD N15 developed a "hot pixel" defect that requires masking out a small region. That problem largely disappeared by February 2018. On Aug. 31, 2018 one half of N10 (\#41) developed a problem (exposure number $>767395$ ) with the serial register that resulted in poor charge transfer efficiency (CTE). On Sept. 4 it was thought to be fixed, but the problem reappeared on Sept. 8 starting with exposure 771163. A script that fixed the problem, when detected, was implemented on Sept. 12. The transition card and cables [6] were replaced on Sept. 24, after exposure 777289. It wasn't a problem to DES after that.

Maintenance and repair of the filter changer was performed shortly after Y 5 to correct a problem, which started prior to Y5, with one of the eight slots. In March 2018 a team of DES and CTIO people found that the two-way compressed air cylinder, which pushed the filter in/out, leaked when trying to push the filter in. That filter slot was replaced. A near disaster happened during this maintenance whereby some of the ball-bearings (BBs) from inside one of the linear bearings fell out when the linear bearing was pulled partway off the bearing rail. We were very lucky that we didn't lose any of the BBs. One of us, Alfredo Zenteno (CTIO), devised a clever method for putting the BBs back into the linear bearing, a procedure generally considered "nigh-impossible". During reinstallation of the FCM onto the camera, one of the exhaust valves that sticks out a little was damaged and that filter slot was made inoperable. A second slot was problematic because of a poor 
adjustment of the air cushion valve that slows insertion before that filter cartridge hits the stop. These were both repaired in early August 2018 by CTIO. The shutter maintenance, consisting of cleaning and lubrication, was performed in March 2018 without incident.

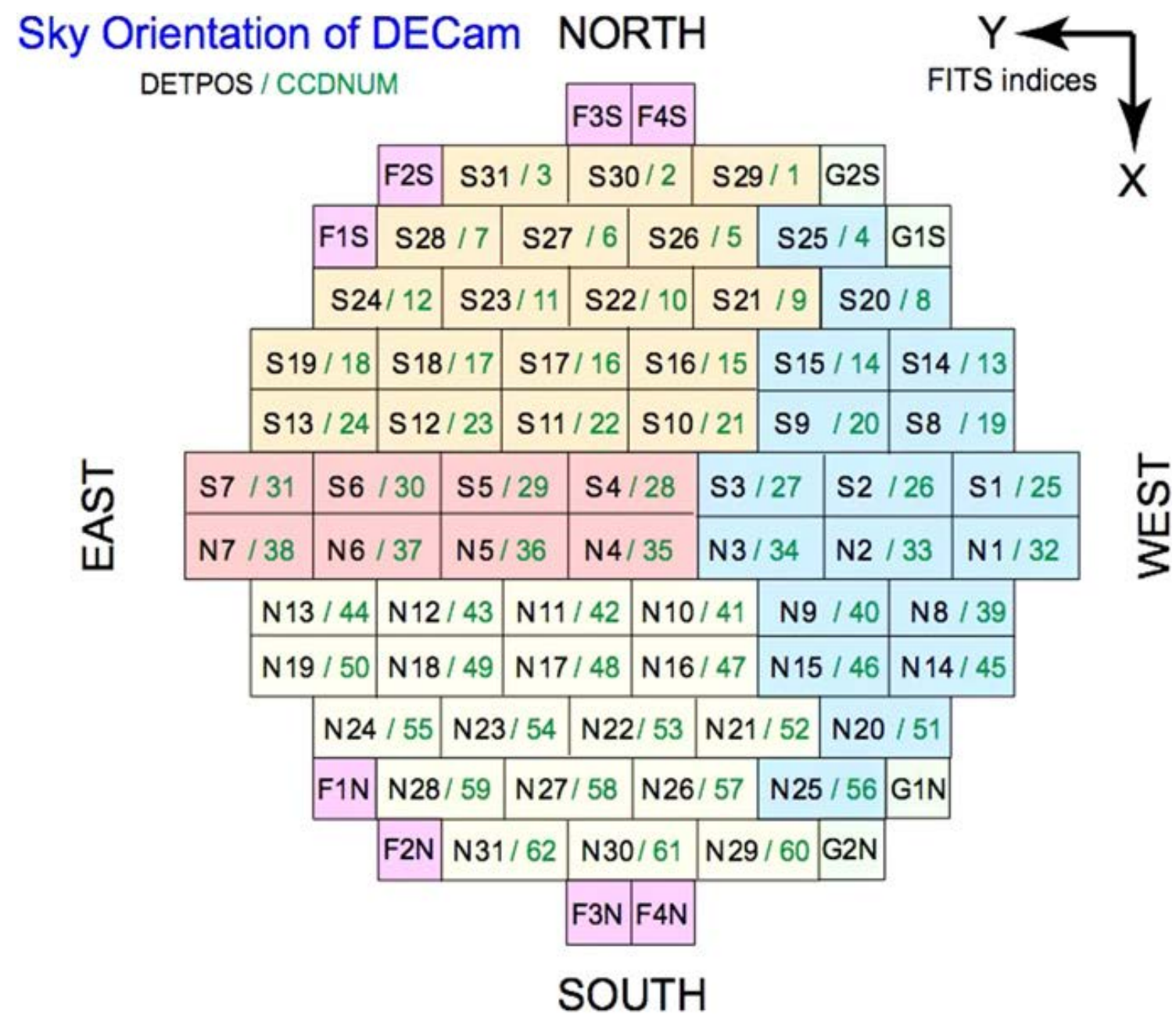

Figure 3 DECam focal plane showing the $622 k \times 4 k C C D s, 82 k \times 2 k C C D s$ (labeled " $F$ ") for the adaptive optics system, and 4 $2 k \times 2 k C C D s$ (labeled " $G$ ") for guiding. The orientation of the sky is indicated. The label (e.g., S30) indicates a position on the focal plane. The label (e.g., 2) indicates the number of the CCD as is in the multi-extension FITS header. When the focal plane is viewed with the real-time display at the telescope or with default SAOImage DS9 settings, the direction labeled "north" is displayed to the left and "east" at the top. The background colors of the CCDs indicate the electronics backplane that reads them out. See Ref. [6] for details.

Finally, re-aluminization of the Blanco primary mirror was sucessfully completed in the period June 19 to July 10, 2018. Previously done in March 2011, the impact was slight loss in reflectivity that amounted to more than 0.2 magnitudes in a 90 second exposure by the end of Y5. The realuminization resulted in improved reflectivity, as shown in Figure 4. In addition, we observe the recovered reflectivity through comparison of flux from stars and galaxies in recent images compared with what we had in last season. 


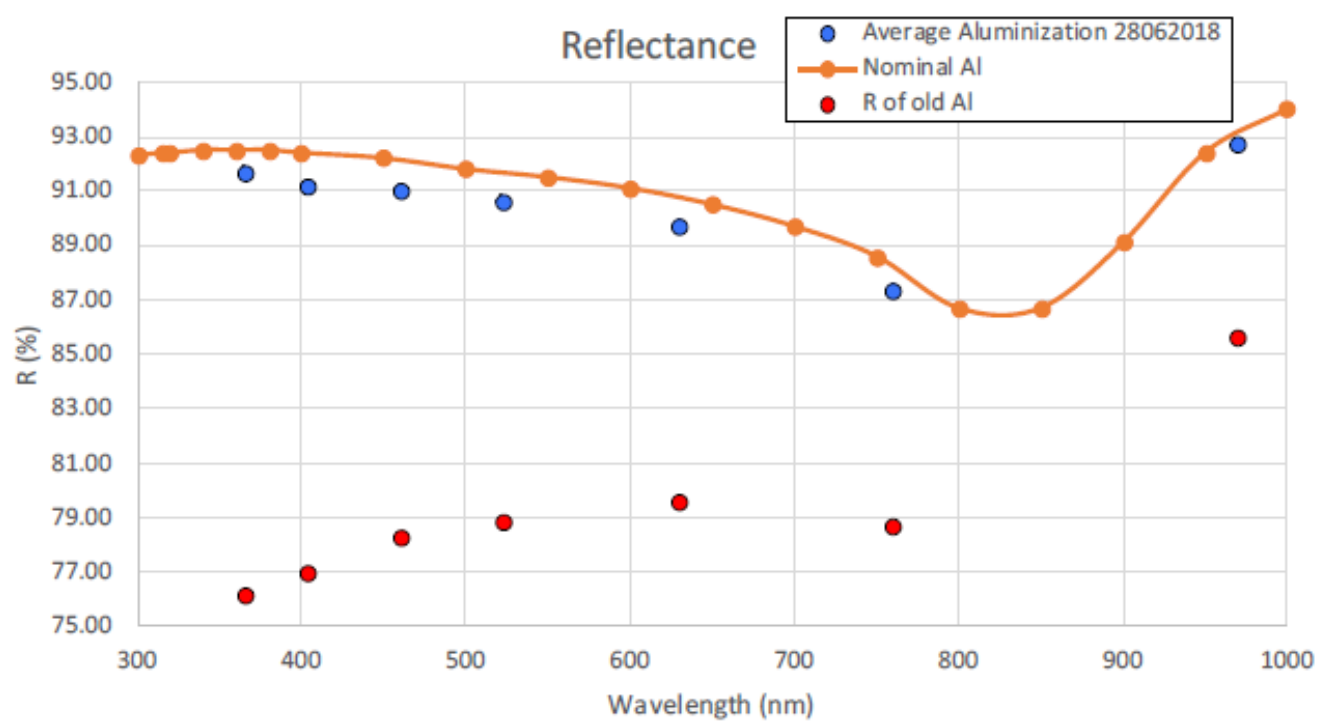

Figure 4. Reflectance as a function of wavelength measured on the Blanco M1 (primary mirror) after new aluminum coating. It can be directly compared to the reflectance values measured before stripping off the old aluminum, as well as to the nominal reflectance of aluminum. Plot is from A. Walker, CTIO.

A record of important events or changes to the camera or telescope is available online [14].

\section{THE DES SURVEY PROCEDURES}

\section{Observation Schedule \& Staffing}

DES observed for 105 night-equivalents averaged over 5 years and for 52 nights during Y6. Table 2 shows the nominal start and end dates for Y1 through Y6 as well as the breakdown into scheduled half-nights and full nights.

As in previous seasons, the DES observations were staffed at the telescope by collaboration members. The Operations Scientist scheduled the observing team from among the volunteers. There were three observing roles during full nights. "Observer 1" controlled the camera through the data-acquisition interfaces [6] and executed the nightly program by following standard DES observing procedures [15]. This observer ensured that images were being recorded, paid attention to the alarms and warnings, and solved routine problems where procedures have been established. "Observer 2" performed quality control procedures, checked the exposures for problems and ensured that the image quality was as expected given the current conditions. The "Run Manager" was the lead observer and was responsible for ensuring that the two other observers understood how to perform their roles. Though observers were asked to read online procedures in advance of their observing trips, training was performed on site by the Run Manager. The Run Manager also had some daytime responsibilities described below, so they were not expected to stay up for the full night. During half-night observations DES usually had only two observers with the Run Manager taking on one of the roles. 
Generally, the "Observer 1" and "Observer 2" roles could be performed by an inexperienced but attentive observer. We sometimes filled those positions with people who have no previous observing experience. We required Run Managers to have mastered both of those observing roles and to have been instructed in their new duties. In practice, by the start of Y4, the observing procedures had been improved and failure probabilities reduced to the point that an attentive rookie observer could be qualified as a "Run Manager" within a few nights of experience. However, we had fewer opportunities for that in Y6. Noting that during Y1 through Y5 we tended to have long blocks of consecutive observing nights (typically spanning 10 to 20 nights), during Y6 there was only one long block of 25 nights. Much of the rest of the Y6 observing was in 4-night long blocks with several night's separations between them. For those short observing blocks we usually had a fixed team cover all the nights, and little opportunity for newly minted Run Managers to train the next set of observers.

Support was available to the observers through the CTIO Telescope Operator (on hand), the CTIO Observer Support Specialist (on-site), the CTIO Instrument Scientist (typically by phone), and the DES Operations Scientist \& Support Team (by internet connection). The CTIO staff handled most of the infrequent observing glitches and stops.

\begin{tabular}{|c|l|l|c|c|c|}
\hline Season & Start Date & End Date & $\begin{array}{c}\mathbf{2}^{\text {nd }} \text { Half } \\
\text { Nights }\end{array}$ & $\begin{array}{c}\text { Full } \\
\text { Nights }\end{array}$ & $\begin{array}{c}\mathbf{1}^{\text {st }} \text { Half } \\
\text { Nights }\end{array}$ \\
\hline Y1 & Aug. 31, 2013 & Feb. 09, 2014 & 0 & 91 & 28 \\
\hline Y2 & Aug. 15, 2014 & Feb. 15, 2015 & 10 & 80 & 41 \\
\hline Y3 & Aug. 04, 2015 & Feb. 12, 2016 & 32 & 73 & 39 \\
\hline Y4 & Aug. 13, 2016 & Feb. 18, 2017 & 19 & 76 & 49 \\
\hline Y5 & Aug. 15, 2017 & Feb. 22, 2018 & 21 & $71^{*}$ & 56 \\
\hline Y6 & Sept. 08, 2018 & Jan. 09, 2019 & 14 & $37^{*}$ & 23 \\
\hline
\end{tabular}

Table 2 Number of scheduled observing nights for each DES observing season. DES was originally scheduled for 525 full-night equivalents over 5 years. That the total of any season might be more or less than 105 full-night equivalents (Y1-Y5) or 52 full-night equivalents (Y6) reflects how DES was compensated for time spent on non-DES TOO's or other programs. For instance, Y4 has 110 scheduled nights, instead of the usual 105. That is because we were expected to expend 5 nights on various TOO programs. Instead, we expended 2 nights. To make up for that, Y5 was originally scheduled for only 104 nights with one of those nights expected to be expended over a number of nights for a TOO. We did that TOO, as well as 30 hours on the DES-GW TOO. Those 30 hours were reimbursed to DES in February 2018, so that we ended up with 102 nights-equivalent for DES. The total number is 760 nights or half-nights. *Four of the nights indicated as full nights were $3 / 4$ nights long. Previous versions of this chart didn't indicate the 3/4-night length nights this way, leading to an undercount in the number of nights on which we were scheduled to observe. On some of the scheduled nights we didn't observe because of weather or the condition of the equipment. In addition, we may have observed on a few nights that we weren't scheduled to observe on, such as "Engineering Nights" where the work has been completed, and nights of "Director's Discretion". We don't indicate those here. Including SV and Y1 to Y6, 182 people observed for DES at least once.

\section{Daily Operations Cycle}

The typical "Daily Cycle" started with the Run Manager's Meeting held daily at 16:00 CTIO time. The Run Manager met by phone with the Operations Scientist \& Support and Data Management (DESDM) Teams. We discussed any technical or procedural problems that occurred during the previous night, provided additional information to DESDM about individual images that might be 
problematic (for example, if the telescope slewed during the image), received the DESDMcalculated data quality (see below) from previous night's imaging, and discussed what to expect from the "Observing Tactician" (OBSTAC) (see next subsection) based on the expected weather conditions. We discussed the special procedures that the observers might need to execute, such as or special calibrations or Target-of-Opportunity (TOO) observations, including the conditions under which they are to be observed. Because we didn't have any Y6 SN program and because we had a long list of special observations for photometric redshift calibration to carry out during the $2^{\text {nd }}$ half of Y6, most Y6 nights required the Run Manager to make on-the-fly decisions, based on the weather and seeing conditions, about what to observe. After this meeting the Run Manager implemented the data quality results from the previous night's observations, updating the Exposure Table so that OBSTAC has up-to-date information on which images, if any, need to be redone.

The period before evening twilight was used for calibrations and to establish the basic functionality of the instrument. An LED system [16] illuminated a flat-field screen attached to the inside of the dome. We took a set of biases and flats in each of the filters. These images are used in the daily calibration. An hour before sunset the telescope operator opens the dome. At minus 10 degree twilight (roughly 40 minutes after sunset) the observers executed three standard star field [17] exposure scripts, one at high airmass $(X=1.65$ to 2.1$)$, one at medium airmass $(X=1.25-1.65)$, and one at low airmass $(\mathrm{X}<1.25)$. The standard star fields are fields of stars with previously calibrated brightnesses in each of the DES filter bands. They are used for characterizing that night's instrumental and atmospheric response (by fitting the observations to a set of "photometric equations") and are an integral part of the photometric calibration for DES. At minus 12 degree twilight (roughly 48 minutes after sunset) the observers began either OBSTAC or special condition observations. These continued during the night until minus 10 degree morning twilight, for standard star observations. Observer 1 controls SISPI and makes sure exposures are being recorded as expected. Observer 2 maintains a watch on the data quality using streamlined image analysis tools such as "Quick Reduce" [6,15] developed by DES-Brazil and KENTools [15].

The observers maintain commentary and notes in an electronic logbook. At the end of each night the observers create two night-summaries. The "CTIO Night Report" lists weather conditions, problems encountered, and the fraction of time lost to each. The "DES Night Summary" provides the narrative of the shift including the expected plan with ephemeris, the conditions, accomplishments, problems encountered, and notes for the DESDM team. A series of automatically generated plots and statistics follows, including transparency, PSF, and ellipticity of stars for each image, a note of any gaps between exposures of greater than 60 seconds, the progress on wide-field and SN surveys, and a list of exposures. This summary provides a concise history of what happened during that particular night.

The images were transferred by the NOAO Data Transport System [18] (DTS) to NCSA/UIUC in Urbana-Champaign, Illinois, usually within 5 minutes after the moment that the shutter closed. Copies of the data are stored in La Serena and at the NOAO Science Archive in Tucson [19]. To conserve both disk space and network bandwidth the DECam data were losslessly FITS tilecompressed [20] using FPACK. Note that both the DESDM pipeline, described below, and the Community Pipeline [21] used a lossy FPACK compression [22]. 
The camera and telescope were returned to a safe condition after observations are finished.

\section{OBSERVING TACTICIAN (OBSTAC)}

During the course of the season a variety of weather, seeing, and sky-brightness (Moon) conditions are expected to occur. A computer application, the DES “Observing Tactician" (OBSTAC) [23,24] used this information to select the highest priority fields to observe during the next short ( $\sim 5$ minute) interval throughout the night. The Y6 OBSTAC decision flowchart is shown in Figure 5. It was very different from previous seasons because we were finishing the final tiling of the survey - at a given time there might be only a few exposures left to complete. It started at the topleft of the chart, using measurement of the point-spread-function (PSF), through automatic application of KENTools on recent exposures. At the beginning of the night that information came from the Standard Star exposures. OBSTAC projected that achieved PSF to what we would have were we observing at zenith in the i-band filter. Then it selected an exposure and put it on the camera exposure queue. And then repeat, keeping a few exposures in the queue.

There were four overall "Tacticians", in order of priority so that the first that is satisfied makes the choice of exposures: "Wide-survey", "Poor seeing", "Fallback", and "Redo". Wide-field ruled when the achieved PSFs and expected signals-to-noise ( $\mathrm{t}$ - eff, see next subsection) were good. We note that even if the Moon has set, we might take z-band exposures because those are the only ones left to do. If the PSF achieved in the recent exposures was worse than 1.6", the Run Manager decided whether or not to switch off OBSTAC control and enable by-hand execution of the "Poor Seeing" json scripts discussed at the 4 PM Meeting. If OBSTAC was continued, the Poor seeing Tactician had control. If that tactician could not identify an exposure, the Fallback Tactician took over next. Interestingly, of 426 times that Fallback was called, it could only find an exposure 282 times. In the remaining 144 cases, Redo selected to retake the lowest available signal to noise exposure. There was always one of those.

There are some details of the OBSTAC decision that remain to be described. The box labeled "select highest priority ..." had only one decision, which was implemented towards the end of Y6. That was whether or not the area of the "Southern Sliver" (see Section 4) was not to be included in the survey. The boxes in the OBSTAC flowchart that follow that are the straightforward identification of a few exposures with the earliest setting time, and then the identification among those, of the one with the smallest telescope slew.

\section{Data Processing \& Data Quality}

The DESDM pipeline [25] performs image detrending and calibration, that we refer to as "First Cut", in order to assess the quality of each exposure with respect to the minimum requirements necessary for DES to obtain its scientific objectives. The procedure follows. The overscan is removed and the bias is subtracted. The image is divided by the mean dome "superflat", made from dome flats over several nights. The CCD crosstalk is removed using a premeasured matrix, a linearity correction applied, fringe and pupil ghost corrections are combined, and a star flat is applied to subsections of each CCD. An astrometric solution for each image is found by comparing to known stellar positions in Gaia DR2[26]. Finally, the point spread function (PSF) is determined by examining the shapes of stellar images and then the position, brightness, and rudimentary shape of objects detected in each image are cataloged. 


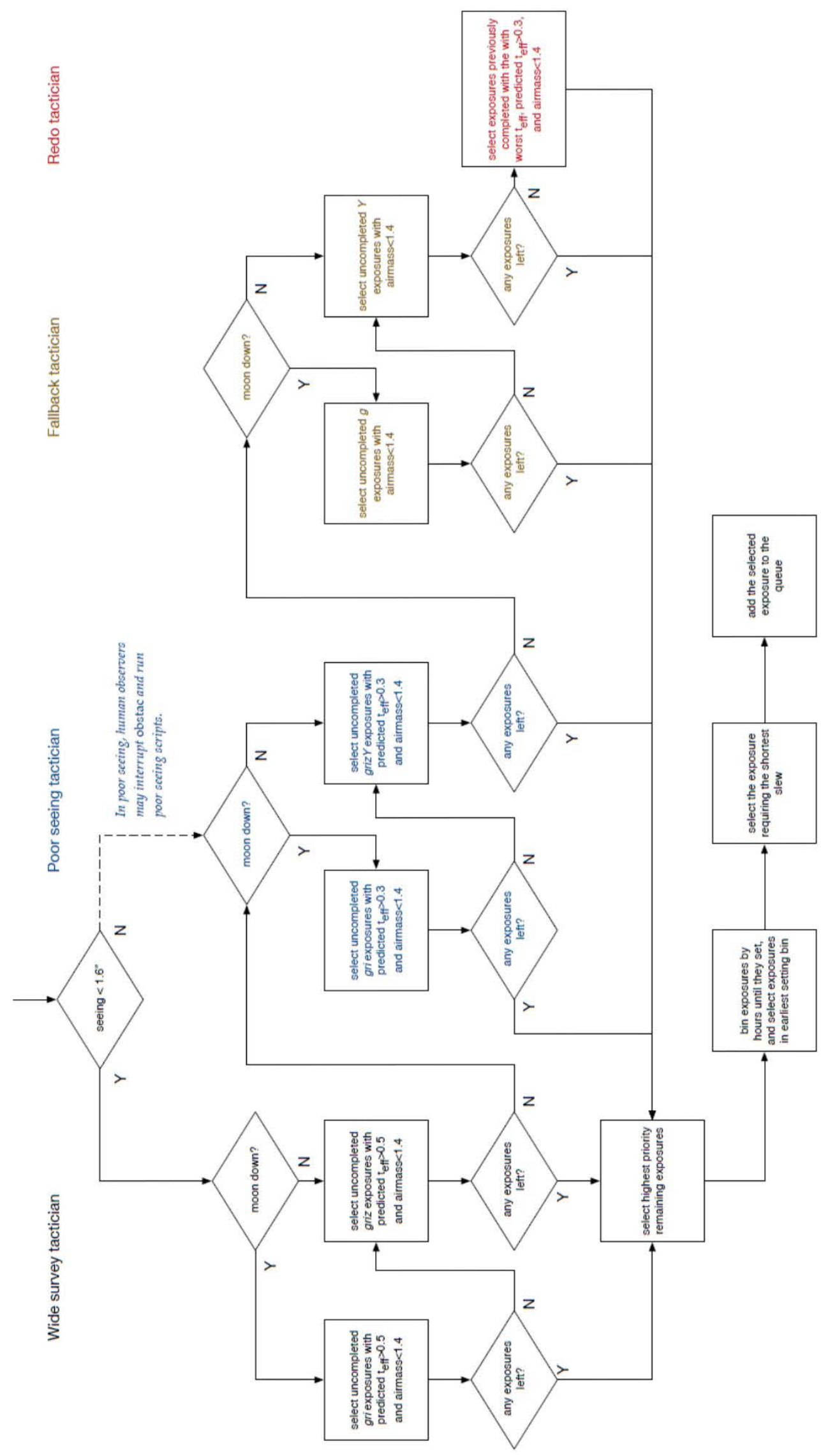


Figure 5 The scheduling algorithm implemented by the Observing Tactician algorithm "OBSTAC" for Y6. We note that in previous seasons we had the SN survey during poor seeing conditions (and that the definition of poor-seeing was different from Y6).

We determine if the image is adequate for the wide-field survey by requiring that the "Effective Exposure Time" [27], $\mathrm{t}_{\text {effective }}=(0.9 \mathrm{k} / \mathrm{FWHM})^{2}\left(\mathrm{Bkgd}_{\text {dark }} / \mathrm{Bkdg}\right)\left(10^{-2 \mathrm{C} / 2.5}\right)$ exceeds a minimum. Here $\mathrm{k}$ is a filter-dependent "Kolmogorov Factor" scaled relative to i-band that takes into account the natural seeing dependence on wavelength, FWHM is the delivered point-spread-function for stars, Bkgd and Bkgd dark are the measured sky background and dark sky condition, and $\mathrm{C}$ is the atmospheric extinction offset calculated from a comparison of the brightness of stars within the image to those in the APASS DR7 and/or NOMAD public catalogs [28-29]. $t_{\text {effective }}=1$ corresponds to an exposure taken at zenith with no moon, clear skies, and nominal seeing, and sky brightness, and atmospheric extinction. We require that $t_{\text {effective }}>0.2$ for $g$, and $\mathrm{Y}$-bands and $>0.3$ for $\mathrm{r}$, $\mathrm{i}$, and z-bands for an exposure to be graded "good" enough to be considered successfully completed. Each image is then checked for artifacts, such as satellites and airplane trails, and these images are flagged.

The First Cut processing and data quality evaluation was typically turned around in less than 24 hours. The results are applied to the Exposure Table by the Run Manager as described previously.

\section{THE DES Y6 NARRATIVE, EFFICIENCY, LOSSES, AND PROGRESS}

\subsection{Survey Progress Y1 through Y5}

After DES seasons Y1 through Y5, we had fallen behind WF survey goals by 0.5 seasons, losing a tenth of a season in Y1 primarily due to problems with the Blanco environmental controls, a tenth of a seaon in Y2 primarily due to below average weather, and 4/10 ${ }^{\text {ths }}$ of a season in Y3 due to the worst weather in the 50 year history of CTIO $[3,4]$. That bad weather was due to a strong El Niño [30]. During Y4 and Y 5 we caught up by a total of about $1 / 10^{\text {th }}$ of a season. Figure 6 shows the number of completed tilings vs RA and DEC of good exposures (as defined above) for each filter as of the end of Y5. In g,r, and z-bands, most of the area is complete over 9 tilings with a region in the center in RA that is complete over only 8 tilings. The i-band, similar, has an $\sim 100$ sq. deg. region for which 10-tilings are finished. The Y-band filter needs to be completed only to 8 tilings - the exposures for tilings 7 and 9 were increased from 45 to 90 seconds duration and tilings $8 \& 10$ dropped. This saved approximately 22 hours of overhead. The cost is that the Yband survey is less uniform than that of the other 4 filters. This kind of plot is called a "completion map". By the end of Y5 we had originally planned to have completed 10 tilings in each filter and would have done so under "median" weather conditions. Instead, at the end of Y5 we are $\sim 1 / 2$ season of observations short of our goal. 


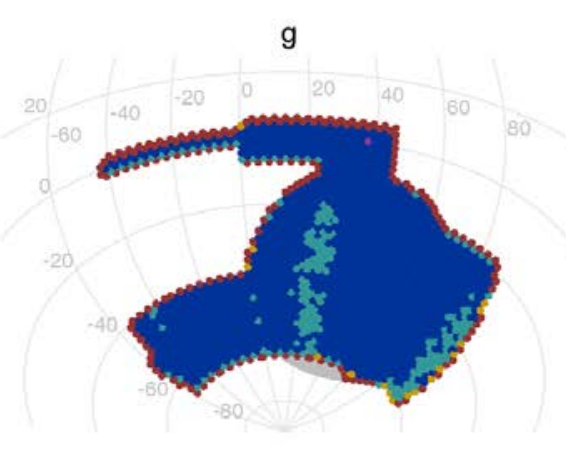

Z

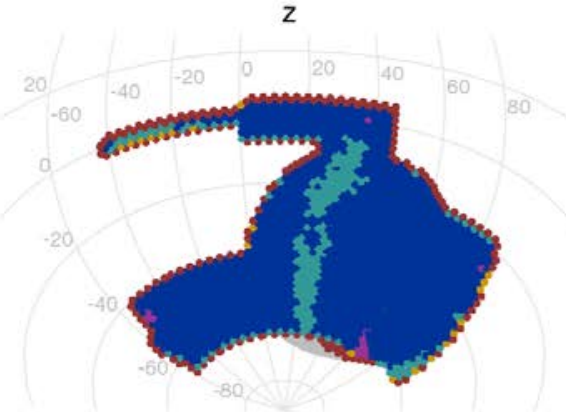

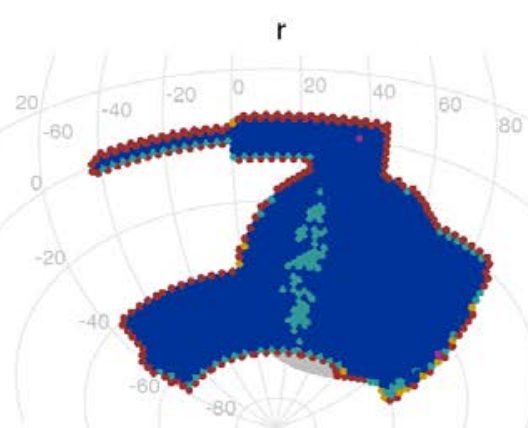

Y

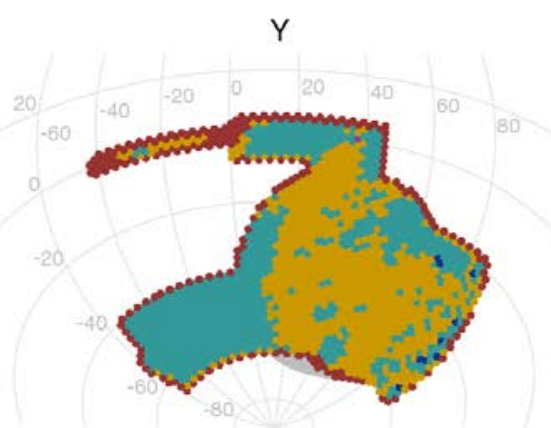

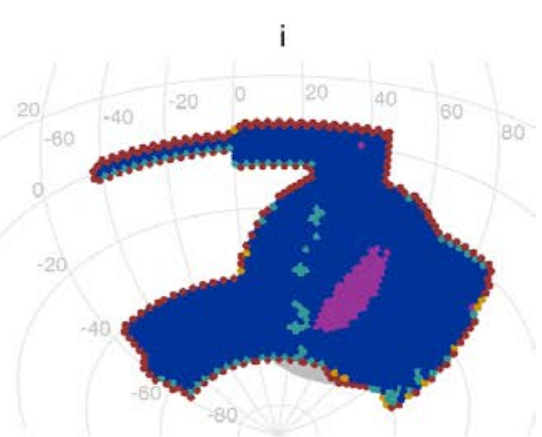

tilings

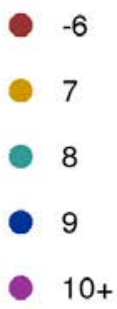

Figure 6 The WF survey completion map at the end of Y5. Given median weather conditions for the survey, by the end of Y5 should have finished the full 10 tilings of the survey. Instead we've finished 9, averaged over the five filters. Some areas are complete (see purple regions for $g, r, i \& z$-band and light blue for Y-band). The completed region in the $i$-band corresponds to where a TOO that required 90 s exposures coincided with the DES footprint.

\subsection{WF Survey Progress in Y6}

Because of Y3's terrible weather, we became newly sensitized to long-term weather predictions and climate conditions. We learned that the Y3 El Niño was caused by the warmer than average temperature of the southern oceans, and it had been predicted by climate forecasters [30]. A few months before the start of Y6, the forecasts predicted El Niño conditions once again.

Y6 started on September 8, 2018 with observing the $2^{\text {nd }}$ half of the nights. We switched to full nights on September 27 and then to $1^{\text {st }}$ half nights on December 8 . We didn't operate the SN program during Y6, so the aim of the observing strategy was simply to complete the wide-field survey, finishing 5000 or more square degrees with good exposures, as described above, in all 10 tilings and all 5 filters. The OBSTAC plan for Y6 was to continue to ensure that exposures on the western side of the WF footprint were given priority at the beginning of the season, because that side of the footprint was already descending. Therefore, OBSTAC prioritized exposures west of R.A. $=30$ degrees by closeness to transit, and east of R.A. $=30$ degrees by setting time. As in Y5, to increase observing efficiency, we removed the thin part of Stripe 82 (west half around DEC $=0$ ) from OBSTAC, preventing our making frequent long slews back and forth between that part of Stripe 82 and the footprint further south. We again used standard observing scripts to observe that part of Stripe 82.

As the survey reached completion, OBSTAC reached states that never occurred in previous years. There were times during which the low priority area in the south ("Southern Sliver", with DEC < $-60 \mathrm{deg}$ ) was the only part of the footprint with uncompleted exposures. Yet there wasn't enough 
observing time left to complete all 10 tilings for that whole region. We modified OBSTAC so that we didn't spend time making observations that would not ultimately contribute to area with 10 tiling depth. The ordering by tiling id was removed. Exposures were prioritized north to south, such that the edge of the footprint spread southward at full depth as additional exposures were added. The requirement that exposures not overlap other overlapping exposures taken in the same night and the same filter was removed. This risked reducing the usefulness of this region for weak lensing but preserved the overall depth.

Finally, when all observable exposures in the wide footprint were complete, OBSTAC would start redoing exposures whose data quality ( $\left.\mathrm{t}_{\mathrm{eff}}\right)$ was only slightly above the data quality cutoff. This rarely occurred, because this time was better used for the ancillary programs (listed below).

We tracked the Y6 observing efficiency using a combination of information including the ELog, the DES and CTIO Night Reports, and other tools. The DES and CTIO Night Reports were collated to produce the estimate of how we spent our time. That is reported in Table 3. Y6 got off to a moderately poor start due to worse-than-average weather during September ( $28 \%$ clouded out) and October (38\% clouded out). But then Y6 turned around with a superb November, with no observations lost to weather and some "best seeing records" in some filters (see Table 4). For the remainder of Y6 the observations lost little time to clouds. The camera and telescope were extremely reliable with only 3 hours lost. Table 3 provides statistics for the use of DES observing time in Y1 through Y6. During Y6 observations the shutter was open $62.4 \%$ of the available time (not clouded out, etc ...). This was $\sim 6 \%$ less than $\mathrm{Y} 4$ and $\mathrm{Y} 5$ and is not surprising because there were no SN exposures in Y6 and because we needed to take exposures in a "fill-in" fashion, resulting in some longer slews than previous in seasons.

In Y6 we recorded 9,036 exposures. Of those $89.1 \%$ were graded good by First Cut, compared to an average of $82.5 \%$ for Y1 to Y5 (though dominated by Y3 at 71\%). Taking into account that a few of these were Y-band tiling 7 or 9 (which should be counted as double), the Y6 total goes to 8,864 good ones. Figure 6 shows the WF survey completion map after Y6. In the last few nights of observing, we built 10-tiling area in the region $20<\mathrm{RA}<60$, DEC $<-60$, which we named "the Southern Sliver". We finished 5,085 sq. degrees to survey depth of 10 tilings, meeting the 5,000 sq. deg. milestone originally planned for the survey.

DES science requirements, particularly the weak lensing shear analyses [31], put a premium value on good point-spread-function (PSF) for the r,i, and z-band exposures. Typical of previous DES seasons, the Y6 data meets the standard that we will need. Figure 8 shows the PSF for WF images Y6. The median PSF of all good images was 0.92 ", 0.03 " worse than Y5. This is perhaps because we cut away from the WF survey to Y6 "poor seeing" programs when the seeing was worse than 1.6", rather than the typical 1.1" (for Y1-Y5) when we had the SN program. 
Table 3 DES Operational efficiency sums accumulated through Y6. These are based on the observer's reports in the CTIO Night Summaries. "Time Available" is the time we should spend observing." Observing Time" is the number of hours the observers were actually engaged in observing. "Engineering Observations" are those in service to the understanding of camera or telescope systematics. Next is indicated the number of hours lost to bad weather that results in closing the dome instead of observing, to a failure of the telescope, dome, or mountaintop infrastructure, to the camera. *The Y1-Y5 weather loss is dominated by Y3, when we were "dome closed" for 294 hours (30.3\%).

\begin{tabular}{|c|c|c|c|}
\hline Operations & $\begin{array}{c}\text { DES Yr. 1-5 } \\
\text { Averages } \\
\text { Hrs. (\%) } \\
\end{array}$ & $\begin{array}{c}\text { DES Yr. } 6 \\
\text { Accumulated } \\
\text { Hrs. }(\%) \\
\end{array}$ & $\begin{array}{c}\text { DES Yr. 1-6 Totals } \\
\text { Hrs. }\end{array}$ \\
\hline $\begin{array}{c}\text { Observing Time } \\
\text { Available }\end{array}$ & 958 & 490.65 & 5279 \\
\hline Time Observing & $\begin{array}{c}798 \\
(83.4 \%) \\
\end{array}$ & $\begin{array}{c}405.4 \\
(82.6 \%) \\
\end{array}$ & $\begin{array}{c}4397 \\
(83.4 \%) \\
\end{array}$ \\
\hline $\begin{array}{c}\text { Engineering } \\
\text { Observations \& Other } \\
\text { Losses }\end{array}$ & $\begin{array}{c}1 \frac{1}{2} \\
(<0.2 \%)\end{array}$ & $\begin{array}{c}2.25 \\
(0.5 \%)\end{array}$ & $\begin{array}{c}93 / 4 \\
(0.2 \%)\end{array}$ \\
\hline Bad Weather & $\begin{array}{c}128 \% \\
(13.4 \% *)\end{array}$ & $\begin{array}{c}80 \\
(16.3 \%)\end{array}$ & $\begin{array}{c}722.6 \\
(13.7 \%)\end{array}$ \\
\hline $\begin{array}{c}\text { Telescope or } \\
\text { Infrastructure Failure }\end{array}$ & $\begin{array}{l}14 \frac{1}{2} \\
(1.5 \%)\end{array}$ & $\begin{array}{c}3 \\
(0.6 \%)\end{array}$ & $\begin{array}{c}75.5 \\
(1.4 \%)\end{array}$ \\
\hline Camera Systems Failure & $\begin{array}{l}14 \% / 4 \\
(1.5 \%)\end{array}$ & $\begin{array}{c}0 \\
(0 \%)\end{array}$ & $\begin{array}{c}73.6 \\
(1.4 \%)\end{array}$ \\
\hline
\end{tabular}

Table 4 Best DES PSF for any exposure for each filter Y1 to Y6. For other "Bests of" see URL https://cdcvs.fnal.gov/redmine/projects/desops/wiki/Bragging_rights_(ie_seeing_records_etc)

\begin{tabular}{|c|c|c|}
\hline Filter & $\begin{array}{c}\text { Best PSF } \\
\text { (arcsec) }\end{array}$ & Date \\
\hline u-band & $0.86 ”$ & Jan. 06, 2018 \\
\hline g-band & $0.72 ”$ & Nov. 26, 2018 \\
\hline r-band & $0.68 ”$ & Nov. 26, 2018 \\
\hline i-band & $0.67 ”$ & Nov. 26, 2018 \\
\hline z-band & $0.63 ”$ & Nov. 05, 2016 \\
\hline Y-band & $0.61 ”$ & Nov. 05, 2016 \\
\hline
\end{tabular}


g

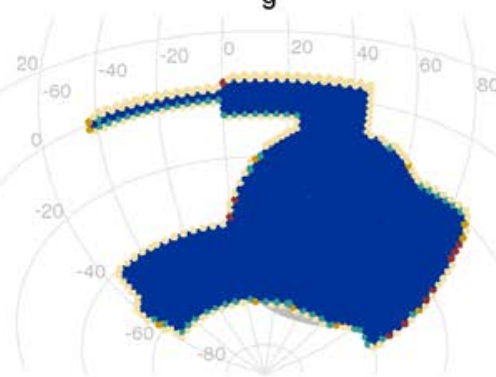

Z

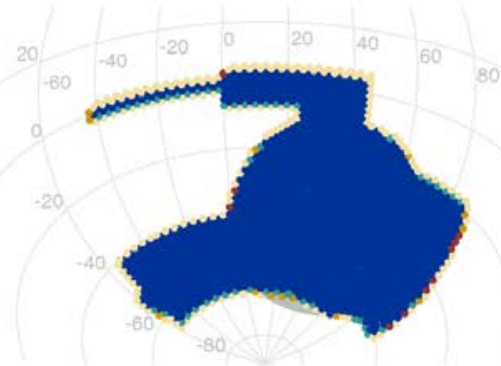

r

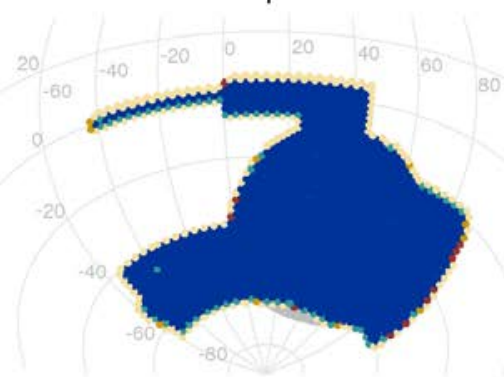

Y

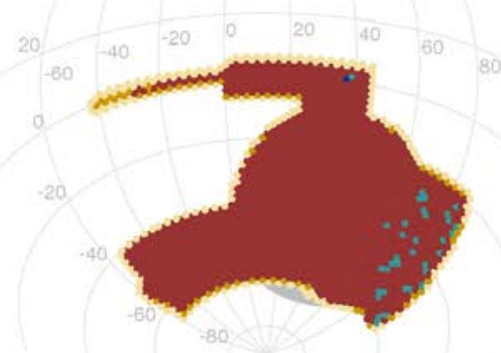

i

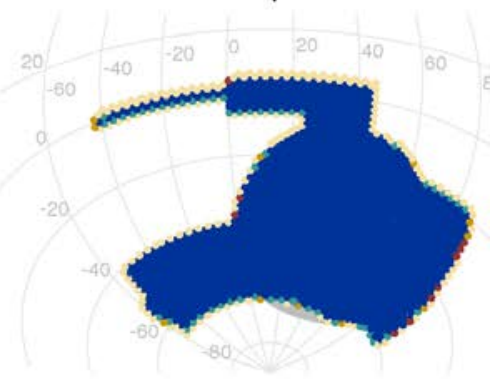

tilings

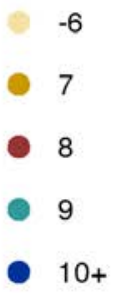

Figure 7 The WF survey completion map at the end of Y6. Apart for a small part of Stripe 82 in the Y-band, all WF survey exposures were completed through 10 tilings (8 for Y-band, with 2 of those being 90s instead of the nominal $45 \mathrm{~s}$ duration). The total area completed was 5,085 sq.-degs. The "Southern Sliver" is roughly from $20<R A<60$ and south of -60 DEC.

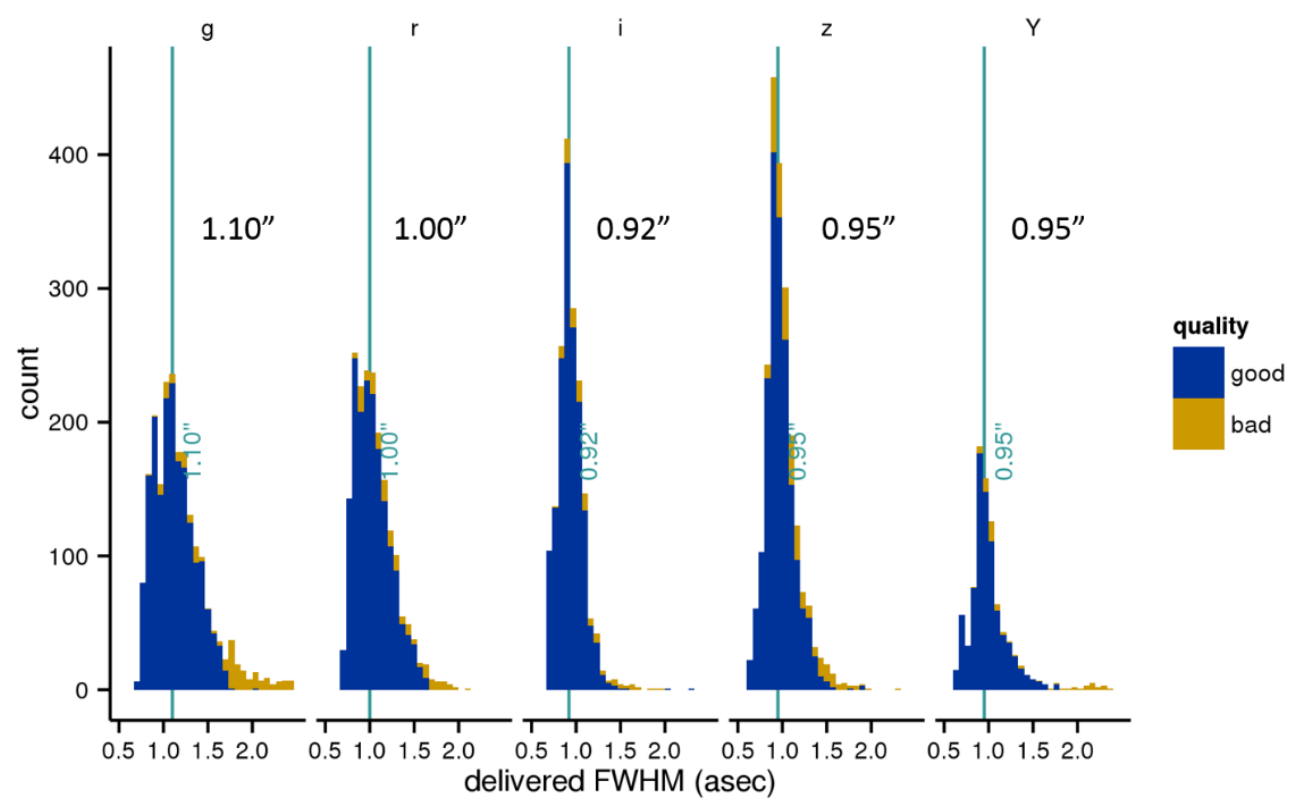

Figure 8 The "point-spread-function" (PSF), full-width half-max, of Y6 DES WF survey exposures. The quality "good" or "bad" is the result of First Cut, discussed in Section 2. The achieved Y6 PSF averages weren't as good as during Y5 but were similar to $Y 4$ and to the other previous seasons. 

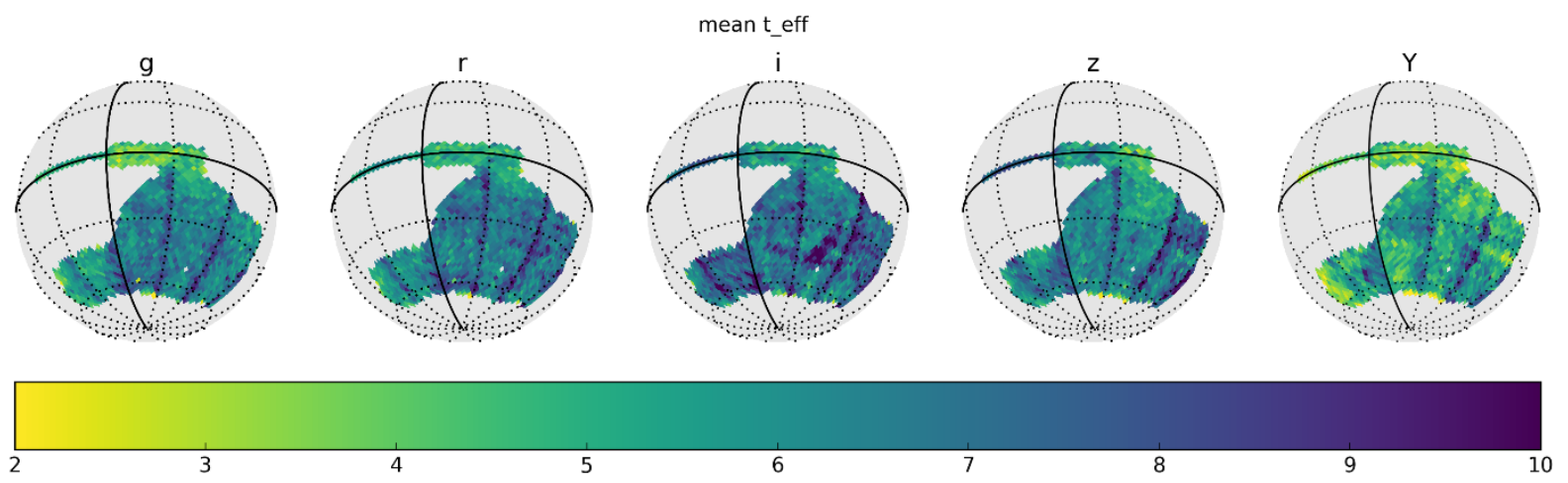

Figure 9 Sum of Teff over the survey in each of the 5 filters after the end of survey observations.

\subsection{Y6 "Poor Seeing" Program}

We didn't operate the SN survey during Y6, so that program was not available when the seeing turned poor. Instead we created "Poor Seeing" programs that were to be hand-executed by the observers. These were typically triggered by conditions resulting in exposures with PSF $>1.6$ " for extended periods. A subsection below describes these programs.

Observing conditions were defined as "Poor seeing" if time periods more than $~ 10$ minutes long resulted in exposures that have PSF $>1.6$ ". The observers would then change from ObsTac to the Poor Seeing Program. The three "poor seeing" programs that we run are called "Photosweep", "Narrowband", and "Reverberation Mapping".

\section{Photo-Sweep}

In the Photosweep program, we attempted to tie together the photometry across the full survey footprint by having DECam visit widely spaced positions across the FOV in a time that we hoped was short compared to the timescale of transparency variations in the atmosphere. This would reduce the possibility of slight gradients in the calibration. The scripts moved the telescope to a series of positions that span all parts of the DES footprint that were then at airmass $<1.8$ and at least 30 degrees from the Moon. At each position, 15-second exposures were taken in the g,r,i,z, and Y-band filters. Then the telescope made a fairly long slew to the next position - somewhere between 15 and 90 degrees. These positions were ordered by azimuth to minimize the dome motion required. Essentially the telescope was loop around the zenith when running the sequence. The scripts were about an hour long and needed to be completed to be useful. Photosweep required photometric conditions.

Going into December we had taken only 35 minutes of photosweeps. It was apparent that we weren't going to get enough of these to be useful for improving the calibration. We gave it more priority, so by mid-December the total was 233 minutes. Wanting still more (and realizing we were going to accomplish the primary goal of completing the wide survey field), we reduced the seeing threshold for photosweep fields to 1.1". Another 138 minutes of photosweeps were taken in those (good) seeing conditions. 


\section{Narrowband}

In the Narrowband program, we obtained imaging in the SN fields having deep NIR data but not yet having images from the narrowband filter centered at $964 \mathrm{~nm}$. We expected that detection of emission lines in this filter could be used to check or improve the $\mathrm{n}(\mathrm{z})$ determinations in these fields, as well as search for strong line-emitting galaxies. We sandwiched the narrowband exposures with z-band exposures in order to veto variable objects. The scripts are nominally an hour long but are useful if truncated so long as we got the z-band data at the beginning and end. We recorded 90 minutes of "poor-seeing" narrowband exposures prior to December. In December we took 90 minutes more of those on the E2 SN field under excellent seeing conditions. We didn't need any more. Narrowband could be taken even if the conditions were not photometric.

\section{Reverberation Mapping}

The "Reverberation Mapping" (RM) program was to use DECam for broadband imaging of the 10 DES SN fields to provide lightcurves that complement the then ongoing spectroscopic monitoring of AGN being done by OzDES. The OzDES collaboration had time on the Australian Astronomical Telescope (AAT) on Sept. 3-6, Oct. 6-8, Nov. 7-9, and Dec. 1-3. We were asked to take DECam observations, which took just under two hours to complete, within about 5 nights of the center of each of those AAT observations. The DECam exposures could be taken if 1.6" > PSF $>2.7$ ", so long as the sky was within about $20 \%$ of photometric. If we were getting close to the end of the window, we were to simply execute the RM program with priority greater than DES regardless of the conditions. So, we did this in cooperation with the DECam RM team (PIMartini). Our schedule was pre-imbursed for the time that we spent executing this program.

\subsection{Target of Opportunity Observing}

For recent observing seasons DES arranged with CTIO and some external group(s) to provide "Target of Opportunity" (TOO) observing time specified by the external group during nights that DES was observing. In case of the need for such observations, the external group contacted DES operations, and provided the observing scripts and the order and conditions under which they were to be executed. The external groups shared weather risk with DES. The total time that was used for TOO's was tracked and reimbursed by CTIO in the A semesters, which start in February of a given year. An array of remote stations set up at Fermilab in the "Neutrino Remote Operations Center" ( $v$ ROC) was sometimes used to participate in and assist with the TOO observations that were going on at CTIO. DES observers smoothly handled more than 40 TOO interruptions of OBSTAC in the previous season.

The Y6 TOO program was "DES-IC" (PI-Bechtol), which sought optical counterparts to energetic neutrino triggers from ICE-CUBE [32-34]. They were allocated 1.5 nights but used very little of it as there were no well-suited alerts.

\subsection{Additional Special Purpose Fields Observed in Y6}

\section{Star Flats}

The CCDs position on the focal plane is set by two INVAR pins in the CCD's foot that are inserted into two holes in the thick aluminum focal plane support plate [6]. The pins are (necessarily) slightly smaller than the holes. We found that tiny shifts of the CCD could occur in the warmupcooldown cycle. After such cycles we measure the positions of the CCDs using well known 
positions of stars. We call these "Star Flats". This is usually been done in "Engineering Time" near the full moon. We used 142 minutes of our observing time taking star-flats during December.

\section{Deep Fields}

In order to improve the photo-z calibration we selected two tiles for creation of new 10x WF survey depth "Deep Fields". Alhambra-2/DEEP2\#4/0228+0 [35,36] and MACSJ0416-24 [37,38] have spectroscopy that is complete to depth greater than the DES WF survey depth. The MACSJ041624 field has j,h,k IR data. The Alhambra field does not. Table 5 shows the time spent on observations of the MACSJ0416 field through the end of the survey.

Table 5 Progress on the new "Deep Field" MACSJ0416 as of the survey. Data quality isn't taken into this accounting. The WF survey tiles are $10 \times 90$ seconds.

\begin{tabular}{|c|c|c|}
\hline MACSJ0416 Filter Band & Single Exposure Time (s) & \# Exposures \\
\hline $\mathbf{u}$ & 360 & 26 \\
\hline $\mathbf{g}$ & 300 & 31 \\
\hline $\mathbf{r}$ & 300 & 35 \\
\hline $\mathbf{i}$ & 300 & 32 \\
\hline $\mathbf{z}$ & 360 & 36 \\
\hline
\end{tabular}

\begin{tabular}{|c|c|c|}
\hline Alhambra Filter Band & Single Exposure Time (s) & \# Exposures \\
\hline $\mathbf{u}$ & 360 & 20 (last year) \\
\hline $\mathbf{g}$ & 360 & 20 \\
\hline $\mathbf{r}$ & 360 & 21 \\
\hline $\mathbf{i}$ & 360 & 22 \\
\hline $\mathbf{z}$ & 360 & 23 \\
\hline
\end{tabular}

\section{THE LAST NIGHT OF OBSERVATIONS}

January 9, 2019 was the last night of DES observing. The CTIO chefs served a fine lunch, including extra-special desserts, and CTIO Director Heathcote and the DES Deputy Director served speeches [39] to the CTIO staff and the DES observers. After lunch the observers [40] participated in a video-tour of the telescope that was shown to scientists and NSF/DOE funding agency representatives at the American Astronomical Society Meeting that was going on in Seattle, WA. The 4 o'clock online meeting was attended by at 25 people and it essentially continued, with people coming and going, until the countdown for the end of the final exposure. The seeing conditions were good and the sky was clear and dark. We took the usual standard stars, long exposures of the Alhambra Deep Field, added area to the Southern Sliver, and redid 18 of the poorer but good WF exposures to improve the survey uniformity. Next we took exposures of NGC 1487. We ended with the Fornax Galaxy Cluster, featuring NGC1365, thus repeating exposures of this spectacular barred-spiral galaxy that were taken on official First Light in September 2012. End of half-night standard stars finished with exposure 810381 at $04: 48 Z$ on January $10^{\text {th }}$. 
The build-up to the end of DES observations is nicely documented at URL \#DESendofnights, which includes a collection of recollections and photographs from the DES observers. ${ }^{2}$ Dr. Rutu Das, one of the DES observing stalwarts, posted a poem there, which we use with her permission.

\author{
Our Favorite Sightings \\ (to the tune of "My Favorite Things") \\ Faraway galaxies, perhaps distorted, \\ Galaxy clusters by massiveness sorted, \\ Transient Type-1a supernovae - \\ These are the objects for which we survey. \\ High-richness clusters and galaxy spirals, \\ Snapshots of Lovejoy that kind of went viral, \\ A trio of galaxies merging in dance - \\ These are exposures that hold us entranced. \\ Constraints on cosmology - the stuff of our dreams! \\ Dwarf planet DeeDee, and bright stellar streams, \\ The first ever visible GW source - \\ These are a few of our favorite scores. \\ When the night wanes, \\ When DECam sleeps, \\ No more data to add - \\ We simply remember these wonderful years, \\ And then the end's not ... so bad. \\ by Rutuparna Das
}

\title{
6. SUMMARY
}

The Dark Energy Survey Collaboration studies the accelerating expansion of the Universe through four complementary techniques. To produce the deep, 5000 square-degree survey and the 30 square-degree time-domain SN survey that are specified by the science goals, the collaboration designed and built the Dark Energy Camera, now operating on the Blanco Telescope at CTIO. We have finished observations, and successfully completed the survey performance metrics. There are no plans for another DES observing season. The Dark Energy Camera will continue to be operated as a community instrument by CTIO. The publications resulting from the Dark Energy Survey are available at URL http://dbweb5.fnal.gov:8080/DESPub/app/PB/pub/pbpublished.

\footnotetext{
${ }^{2}$ https://www.darkenergysurvey.org/desendofnights/
} 


\section{ACKNOWLEDGEMENTS}

We are grateful for the extraordinary contributions of our CTIO colleagues and the DES Camera and Operations Support teams in achieving the excellent instrument and telescope conditions that have made this work possible. We are grateful for the administrative and organizational assistance provided by Connie Lang (FNAL) and Ximena Herreros (CTIO).

Funding for the DES Projects has been provided by the U.S. Department of Energy, the U.S. National Science Foundation, the Ministry of Science and Education of Spain, the Science and Technology Facilities Council of the United Kingdom, the Higher Education Funding Council for England, the National Center for Supercomputing Applications at the University of Illinois at Urbana-Champaign, the Kavli Institute of Cosmological Physics at the University of Chicago, the Center for Cosmology and Astro-Particle Physics at the Ohio State University, the Mitchell Institute for Fundamental Physics and Astronomy at Texas A\&M University, Financiadora de Estudos e Projetos, Fundação Carlos Chagas Filho de Amparo à Pesquisa do Estado do Rio de Janeiro, Conselho Nacional de Desenvolvimento Científico e Tecnológico and the Ministério da Ciência, Tecnologia e Inovação, the Deutsche Forschungsgemeinschaft and the Collaborating Institutions in the Dark Energy Survey.

The Collaborating Institutions are Argonne National Laboratory, the University of California at Santa Cruz, the University of Cambridge, Centro de Investigaciones Energéticas, Medioambientales y Tecnológicas-Madrid, the University of Chicago, University College London, the DES-Brazil Consortium, the University of Edinburgh, the Eidgenössische Technische Hochschule (ETH) Zürich, Fermi National Accelerator Laboratory, the University of Illinois at Urbana-Champaign, the Institut de Ciencies de l'Espai (IEEC/CSIC), the Institut de Física d'Altes Energies, Lawrence Berkeley National Laboratory, the Ludwig-Maximilians Universität München and the associated Excellence Cluster Universe, the University of Michigan, the National Optical Astronomy Observatory, the University of Nottingham, The Ohio State University, the University of Pennsylvania, the University of Portsmouth, SLAC National Accelerator Laboratory, Stanford University, the University of Sussex, Texas A\&M University, and the OzDES Membership Consortium.

Based in part on observations at Cerro Tololo Inter-American Observatory, National Optical Astronomy Observatory, which is operated by the Association of Universities for Research in Astronomy (AURA) under a cooperative agreement with the National Science Foundation.

The DES data management system is supported by the National Science Foundation under Grant Numbers AST-1138766 and AST-1536171. The DES participants from Spanish institutions are partially supported by MINECO under grants AYA2015-71825, ESP2015-66861, FPA201568048, SEV-2016-0588, SEV-2016-0597, and MDM-2015-0509, some of which include ERDF funds from the European Union. IFAE is partially funded by the CERCA program of the Generalitat de Catalunya. Research leading to these results has received funding from the European Research Council under the European Union's Seventh Framework Program (FP7/20072013) including ERC grant agreements 240672, 291329, and 306478. We acknowledge support the Brazilian Instituto Nacional de Ciencia e Tecnologia (INCT) e-Universe (CNPq grant 465376/2014-2). 
This manuscript has been authored by Fermi Research Alliance, LLC under Contract No. DEAC02-07CH11359 with the U.S. Department of Energy, Office of Science, Office of High Energy Physics. The United States Government retains and the publisher, by accepting the article for publication, acknowledges that the United States Government retains a non-exclusive, paid-up, irrevocable, world-wide license to publish or reproduce the published form of this manuscript, or allow others to do so, for United States Government purposes.

\section{REFERENCES}

[1] Flaugher, B., "The Dark Energy Survey", Int. J. Modern. Phys. A20, 3121 (2005).

[2] Dark Energy Survey Collaboration, "The Dark Energy Survey: more than dark energy - an overview", MNRAS 460, 1270 (2016).

[3] Diehl, H. T. et al., "Dark Energy Survey Operations: Year 1", Proc. SPIE 9149, 91490V (2014).

[4] Diehl, H. T. et al., "Dark Energy Survey Operations: Years 1 to 3", Proc. SPIE 9910, 99101D (2016).

[5] Diehl, H. T. et al., "Dark Energy Survey Operations: Years 4 and 5", Proc. SPIE 10704, 107040D (2018).

[6] Flaugher, B., Diehl, H. T., Honscheid, K. et al., "The Dark Energy Camera", AJ 150, 150 (2015).

[7] Lewis, P. M., Rogers, H., \& Schindler, R. H., "A radiometric all-sky infrared camera (RASICAM) for DES/CTIO", Proc. SPIE, 7735, 77353C (2010).

[8] Reil, K., Lewis, P., Schindler, R. H., \& Zhang, Z., "An update on the status and performance of the Radiometric All-Sky Infrared Camera (RASICAM)", Proc. SPIE, 9149, 91490U (2014).

[9] Li, T., DePoy, D. L., Kessler, R. et al., "aTmcam: a simple atmospheric transmission monitoring camera for sub $1 \%$ photometric precision", Proc. SPIE, 8446, 84462L (2012)

[10] Li, T., DePoy, D. L., Marshall, J. L. et al., "Monitoring the atmospheric throughput at Cerro Tololo InterAmerican Observatory with aTmCam", Proc. SPIE, 9147, 91476Z (2014)

[11] Ruhl, J. E., et al., "The South Pole Telescope", Proc. SPIE 5498, 5498-11 (2004).

[12] L. Jiang et al., "The Sloan Digital Sky Survey STRIPE 82 Imaging Data: Depth-optimized co-adds over 300 deg $^{2}$ in five filters", ApJS 213, 12 (2014).

[13] Kessler, R., Marriner, J., Childress, M. et al., "The Difference Imaging Pipeline for the Transient Search in the Dark Energy Survey", AJ 150, 172 (2015).

[14] https://cdcvs.fnal.gov/redmine/projects/desops/wiki/Log of Configuration_Changes

[15] See URL https://cdevs.fnal.gov/redmine/projects/desops/wiki

[16] Rheault, J.-P., Depoy, D. L., Marshall, J. L., et al., "Spectrophotometric calibration system for DECam", Proc. SPIE 8446, 84466M (2012).

[17] Tucker, D. L., et al., in ASP Conf. Ser. Vol. 364, The Future of Photometric, Spectrophotometric and Polarimetric Standardization, Astron. Soc. Pac., ed. Sterken C., San Francisco, 187 (2007).

[18] Fitzpatrick, M. J., "DTS: The NOAO Data Transport System”, Proc. SPIE 7737, 77371T (2010).

[19] http://portal-nvo.noao.edu

[20] Pence, W. D., Seaman, R., \& White, R. L., "Lossless Astronomical Image Compression and the Effects of Noise", PASP 121, 414 (2009) and http://heasarc.nasa.gov/fitsio/fpack.

[21] Smith, R. C., Walker, A. R., and Miller, C., "DECam Community Pipeline Software requirements and technical Specifications", http://www.noao.edu/meetings/decam/media/community pipeline.pdf.

[22] Pence, W. D., White, R. L., \& Seaman, R., "Optimal Compression of Floating-point Astronomical Images without Significant Loss of Information", PASP 122, 1065 (2010).

[23] Neilsen, E., and Annis, J., "OBSTAC: automated execution of Dark Energy Survey observing tactics", Astronomical Data Analysis Software and Systems XXIII Proceedings. Edited by N. Manset and P. Forshay ASP conference series, vol. 485, 77 (2014). FERMILAB-CONF-13-397-CD.

[24] Neilsen, E. "Dark Energy Survey's Observation Strategy, Tactics, and Exposure Scheduler", FERMILAB Technical Memo TM-2714-AE-CD-PPD

[25] Morganson, E. et al., "The Dark Energy Survey Image Processing Pipeline”, PASP 130, 989 (2018).

[26] Brown, A. G. A. et al., "Gaia Data Release 2: Summary of the contents and survey properties", A\&A 616, A1 (2018). 
[27] Neilsen, E., Bernstein, G., Gruendl, R., and Kent, S., "Limiting magnitude, $\tau$, teff, and image quality in DES Year 1", FERMILAB-TM-2610-AE-CD (2015).

[28]Hendon, A. A., et al., JAAVSO 40, 430 (2012) and http://www.aavso.org/apass.

[29] http://www.ap-i.net/skychart/en/news/nomad.

[30] http://iri.columbia.edu/our-expertise/climate/forecasts/enso/current/

[31]Zuntz, J. et al., "Dark Energy Survey Year 1 Results: Weak Lensing Shape Catalogues", MNRAS 481, 1149 (2018)

[32] https://icecube.wisc.edu/

[33] Morgan, R., Bechtol, K., Kessler, R. and Herner, K. in "A DECam Search for Explosive Optical Transients in Association with IceCube Neutrino Alerts", in MultiMessenger MiniSymposium at APS April Meeting 2019 vol. 64, \#3 (2019).

[34] Morgan, R. et al., "A DECam search for explosive optical transients associated with IceCube neutrinos", submitted.

[35] Newman, J. A. et al, "The DEEP2 Galaxy Redshift Survey: Design, Observations, Data Reduction, and Redshifts", ApJS 208, 5 (2013).

[36]http://deep.ps.uci.edu/

[37] Ebeling, H., Ma, C.-J., and Barrett, E., "Spectroscopic Redshifts of Galaxies within the Frontier Fields", ApJS 211, 21 (2014).

[38] Balestra, I. et al., "CLASH-VLT: Dissecting the Frontier Fields Galaxy Cluster MACS J0416.1-2403with 800 Spectra of Member Galaxies", ApJS 224, 33 (2016).

[39]DES Last Night Ceremonial Luncheon Speech: https://www-d0.fnal.gov/ diehl/Public/DES-LNO-Speech.html \& http://www.ctio.noao.edu/noao/node/15149

[40] The DES observers at CTIO for "last night" were Brenna Flaugher, Alistair Walker, Marcelle Soares-Santos, Klaus Honscheid, and Tom Diehl. They handed the telescope off at the night's midpoint to DES collaborator Tim Abbott, who was $2^{\text {nd }}$ half observer for a different project. 